\title{
Bragg-case x-ray dynamical diffraction propagator in SRW: application to thin crystal phase retarders
}

John Sutter, Oleg Chubar, Alexey Suvorov, Christie Nelson, Kawal Sawhney

John P. Sutter, Oleg Chubar, Alexey Suvorov, Christie Nelson, Kawal Sawhney, "Bragg-case x-ray dynamical diffraction propagator in SRW: application to thin crystal phase retarders," Proc. SPIE 11493, Advances in Computational Methods for X-Ray Optics V, 114930V (21 August 2020); doi: $10.1117 / 12.2567341$

SPIE. Event: SPIE Optical Engineering + Applications, 2020, Online Only 


\title{
Bragg-case X-ray dynamical diffraction propagator in SRW: application to thin crystal phase retarders
}

\author{
John P. Sutter*a, Oleg Chubar ${ }^{\mathrm{b}}$, Alexey Suvorov ${ }^{\mathrm{b}}$, Christie Nelson $^{\mathrm{b}}$ and Kawal Sawhney ${ }^{\mathrm{a}}$ \\ ${ }^{a}$ Diamond Light Source, Harwell Science and Innovation Campus, Chilton, Didcot OX11 9RE,

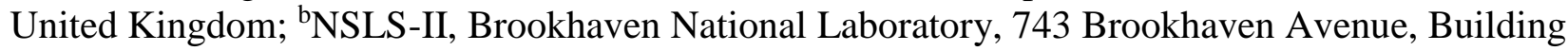 \\ 743, Upton, NY 11973-5000, USA
}

\begin{abstract}
A Bragg-case X-ray dynamical diffraction propagator has already been integrated into the "Synchrotron Radiation Workshop" (SRW) physical optics simulation software package. Previous benchmarking tests on crystal monochromators assumed thicknesses many times the extinction length, for which transmission is negligible. This paper reports tests of this propagator applied to thin crystals in transmission. The chosen example is a phase retarder, which allows users to alter the polarization of an X-ray beam. Phase retarders are often applied to studies of solid-state materials with hard X-rays, which current medium-energy storage ring synchrotron sources typically produce only with linear polarization. Correct designs of phase retarders require the accurate determination of both the intensity and the phase of the diffracted wave in all polarization states. First, to approximate an incident plane wave, SRW is used to simulate the passage of a Gaussian beam of very large radius of curvature through the phase retarder. Then, the phase retarder's effects on a typical undulator beam are simulated and the results are compared. Because X-ray phase retarders are highly sensitive to angular alignment, tolerances in misalignment are also determined. SRW simulations are compared with experimental data from the Integrated In Situ and Resonant Hard X-ray Studies (ISR) beamline at NSLSII. The design of phase retarders can therefore be optimized for X-ray beamlines that must combine variable polarization with focusing or other properties.
\end{abstract}

Keywords: SRW, X-ray, Bragg, crystal, phase retarder, phase plate, physical optics, dynamical diffraction

\section{INTRODUCTION}

\subsection{Motivation and current status}

Diffracting perfect crystals are a mainstay of hard X-ray beamlines at synchrotrons and free electron lasers. The diffraction of X-rays through perfect crystals is governed by the dynamical theory, which has been reviewed by Zachariasen ${ }^{1}$, Batterman and Cole $^{2}$, Authier ${ }^{3}$, and Shvyd ${ }^{\prime} \mathrm{ko}^{4}$. The ray tracing software packages McXTrace ${ }^{5}$, RAY ${ }^{6}$ and SHADOW $^{7}$ have long included the calculation of X-ray reflectivity using the equations of dynamical diffraction for plane waves. However, if the X-ray beam is highly coherent, as on long synchrotron beamlines and free electron lasers, then ray tracing is no longer appropriate; one must use physical optics instead to describe the propagation of the wavefront. Despite the common use of diffracting perfect crystals and the well-known dynamical theory that describes them, software packages that can calculate X-ray wavefront propagation through diffracting perfect crystals remain scarce. The authors of $\mathrm{PHASE}^{8}$, a program written to calculate wavefront propagation through synchrotron beamlines, have proposed to include crystal optics ${ }^{9}$, but in $\mathrm{SRW}^{10}$ a module written to calculate diffraction from perfect crystals in the Bragg (reflection) geometry is now available ${ }^{11}$. The latest Python version of SRW, which includes this module, can be downloaded from Github (https://github.com/ochubar/SRW). SRW can also be executed in the open-source cloudbased interface Sirepo ${ }^{12}$ (https://www.sirepo.com/srw) and is one member of the bundle of software packages in OASYS $^{13}$, which can also be downloaded from Github (https://github.com/oasys-kit).

The benchmarking of the SRW Bragg reflection propagator was performed on crystals whose thickness was many times larger than the Bragg reflection's extinction length so that transmission through the crystal was negligible ${ }^{11}$. This is usually the case for crystal monochromators in the Bragg geometry. However, if a diffracting crystal in the Bragg geometry is sufficiently thin or if its absorption is sufficiently low, it may transmit a significant portion of the incident wavefront. This "Bragg transmission" has not been as widely exploited as Bragg reflection, but in recent years, X-ray

*john.sutter@diamond.ac.uk; phone 441235 778626; fax 44 1235 778784; www.diamond.ac.uk 
interferometers ${ }^{14}$ and an energy analyzer of ultra-high sub-meV resolution ${ }^{15}$ have relied on its special properties, and most of all, X-ray phase retarders have been constructed according to its principles. Therefore, this paper extends the SRW module for X-ray diffraction from perfect crystals in Bragg geometry to Bragg transmission, using phase retarders as an example of practical interest.

Phase retarders alter the polarization of the beam that passes through them. For example, a quarter-wave retarder switches the beam's polarization between linear and circular, and a half-wave retarder rotates the beam's plane of polarization. Because current sources of X-rays with energy $>5 \mathrm{keV}$ at medium-energy synchrotrons produce only horizontally polarized beam, phase retarders are vital for measurements of electronic order, which require linear polarization of variable direction, and for X-ray circular dichroism. Therefore, they are widely used at many synchrotron beamlines, such as the Materials and Magnetism Beamline I16 of Diamond Light Source and the Integrated In Situ and Resonant Hard X-ray Studies (ISR) beamline at NSLS-II. Such practical utility is one good reason for demonstrating phase retarders in SRW, but there are two others. One is complexity: the design of most crystal optics is determined only by the intensity they diffract, but the design of a phase retarder demands accurate calculations of both intensity and phase of the diffracted wave. The other is the question of their compatibility with beam focusing, since X-ray phase retarders are sensitive to the angle between the diffracting net planes and the incident beam, and therefore are affected by the increased divergence that beam focusing introduces.

\subsection{Development and operation of $\mathrm{X}$-ray phase retarders}

A phase retarder must be highly transparent and have a strongly anisotropic refractive index. For visible light, certain crystals such as quartz, calcite, and mica possess these useful properties. For X-rays, however, the refractive index of essentially all materials differs by only a few parts per million from that of vacuum when the beam is simply transmitted, and the absorption is often significant. The way around this lies in Molière's realization that perfect crystals become birefringent close to a Bragg reflection, showing different refractive indices for polarization perpendicular to the "diffraction plane" spanned by the incident and reflected beams ( $s$-polarization) and for polarization parallel to this diffraction plane ( $p$-polarization) ${ }^{16}$. Baranova and Zel'dovich ${ }^{17}$ calculated the birefringence close to a single Bragg reflection, as well as both birefringence and gyrotropy (rotation of the polarization) when two Bragg reflections are simultaneously close to excitation. Because the degree and type of birefringence are highly sensitive to the beam's incidence angle on the crystal, a user can rapidly vary the output polarization by a very small rotation of the crystal. Skalicky and Malgrange ${ }^{18}$ proved experimentally that perfect crystals near a Bragg reflection could indeed function as phase retarders. The effectiveness of X-ray phase retarders at synchrotron beamlines was demonstrated at the Cornell high-energy synchrotron source by Golovchenko et $a l^{19}$, and a quarter-wave retarder was first used to measure circular magnetic X-ray dichroism at the LURE synchrotron ${ }^{20}$. The first two experiments were performed with crystals in Laue geometry, while the third was performed with a crystal in Bragg transmission. Lang and Srajer ${ }^{21}$ compared the practical advantages of Laue and Bragg phase retarders, and found that Bragg transmission was the best choice because it allows energy tunability and helicity switching without the need to collimate the incident beam with special optical components. $\mathrm{X}$-ray phase retarders have been made from both silicon and diamond. Silicon can be manufactured in large ingots with extremely high purity and crystalline perfection, but diamond absorbs X-rays much less strongly. Diamond, therefore, is the material of choice and is sufficient if the incident beam can fit inside a single crystallographically perfect volume, as is usually the case at undulator beamlines. Bragg transmission phase retarders always operate outside the region of total reflection. When accepting a linearly polarized incident beam, they convert the polarization most efficiently when their diffracting net planes are rotated about the beam direction by $45^{\circ}$ so that the $s$ and $p$ components are equal, as shown in Figure 1.

\subsection{Summary of benchmarking tests of SRW Bragg transmission module}

The SRW Bragg transmission module is tested in three ways. First, the phase retarder is simulated by SRW in isolation when illuminated by a linearly horizontally polarized Gaussian beam of very large radius of curvature and hence low divergence, which may be treated as a quasi-plane wave. The total transmitted intensity, and its linearly vertically polarized and right circularly polarized components, are checked against analytical calculations derived from plane-wave dynamical diffraction theory. They are expected to agree well. Second, as a more realistic but still simple test, the phase retarder is included in an SRW simulation of the NSLS-II ISR beamline, which includes an undulator, a double-crystal monochromator, and a pair of focusing KB mirrors. Only the radiation from a single electron is used, and all optical components are ideal. These assumptions are made not only for simplicity, but also to see more clearly how and why the beam divergence affects the polarization conversion and the shape of the focal spot. This SRW simulation of the phase retarder in a beamline also opens the way to more complex simulations that take electron beam emittance and optical 
imperfections into account. Third, experimental measurements of the intensity converted to linear vertical polarization at various rotation angles $\chi$ are compared with both analytical formulas from plane-wave dynamical diffraction and with the SRW beamline simulation previously described. Discrepancies help to determine if the phase retarder is misaligned.

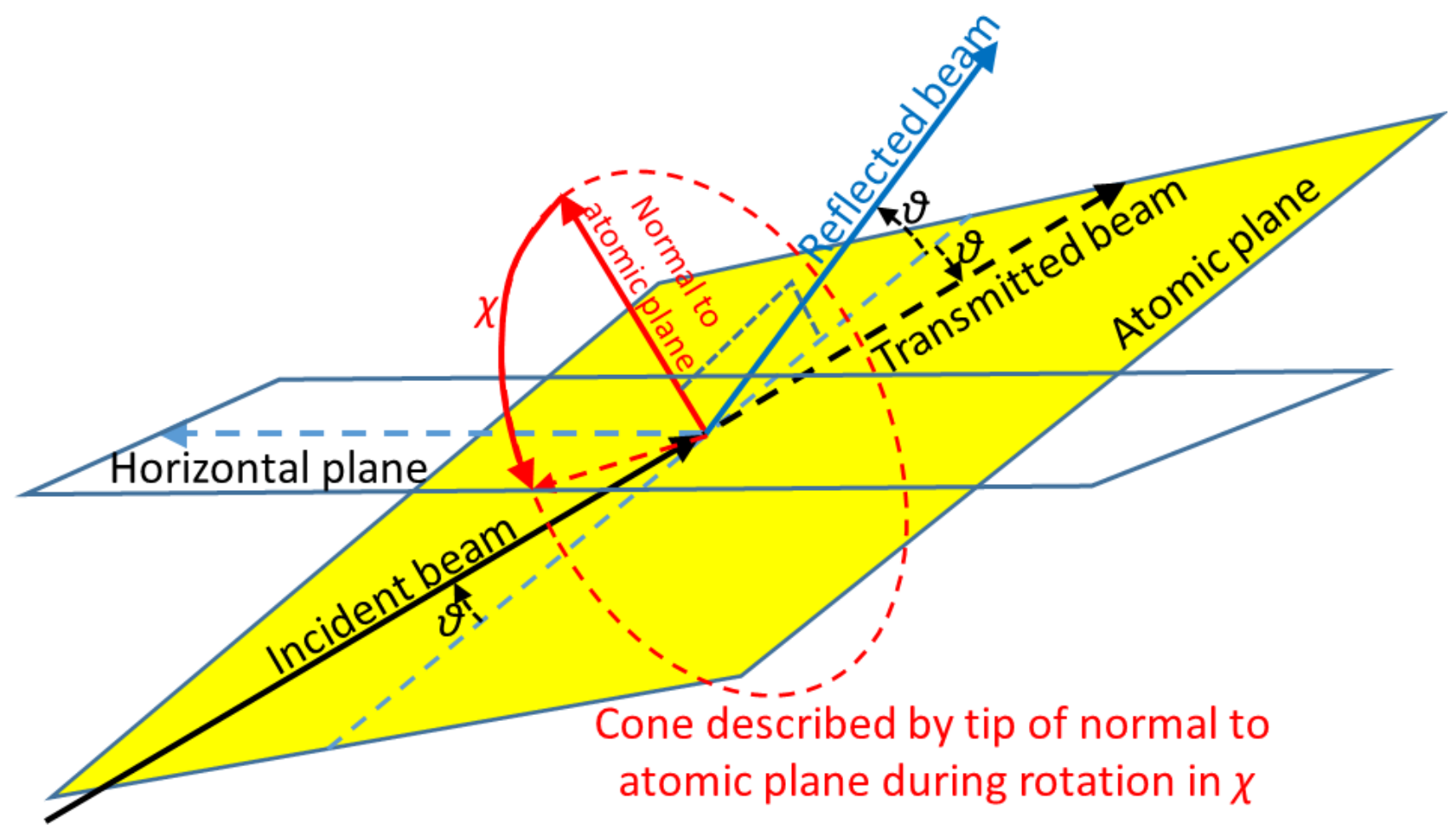

Figure 1. Angles of rotation of phase retarder. $\theta$ sets the Bragg angle. $\chi$ determines the efficiency of the polarization conversion. The "diffraction plane" is the plane spanned by the incident beam and the reflected beam. When $\chi=0$, the normal to the atomic plane lies in the horizontal plane. Phase retarders are operated at $\chi= \pm 45^{\circ}$, where the polarization conversion is optimal.

\section{RESULTS AND DISCUSSION}

\subsection{Material parameters}

The phase retarders were made of diamond. The form factors were calculated from the fits of Waasmaier and Kirfel ${ }^{22}$. Anomalous dispersion corrections were determined by linear interpolation between NIST's tabulated values at https://physics.nist.gov/PhysRefData/FFast/html/form.html ${ }^{23}$. The temperature was $300 \mathrm{~K}$. The Debye-Waller factor was calculated using the Debye model with $\Theta_{M}=1880 \mathrm{~K}$ as measured by Schoening and Vermeulen ${ }^{24}$. A lattice constant of $3.56712 \AA$ and a thermal expansion coefficient of $1.06 \times 10^{-6}$ were provided for a temperature of $298 \mathrm{~K}$ by Stoupin and Shvyd' $\mathrm{ko}^{25}$.

\subsection{First benchmark: quasi-plane wave}

If the incident wave is planar and the crystal is unbounded, the results for intensity and output polarization can be computed from the analytical equations derived from dynamical diffraction theory as in Giles et $a l^{20}$. In an SRW simulation, the incident wave must be spatially bounded, but an approximation to a plane wave can be obtained by using a monochromatic, horizontally linearly polarized Gaussian of large cross section. In this simulation, the Gaussian source has a root mean square source size of $600 \mu \mathrm{m}$ in both the horizontal and the vertical directions. One of the two symmetric (111) diamond phase retarders used at the ISR beamline of NSLS-II is placed $36.2 \mathrm{~m}$ downstream from the source. These phase retarders, along with the photon energy and the radius of the incident wavefront, are given in Table 1. Because the radius of the wavefront at the entrance of each phase retarder is very large, the wavefront can indeed be considered essentially flat. Figure 2 compares the transmission of a quasi-plane wave of $8708 \mathrm{eV}$ through the $510 \mu \mathrm{m}$ thick phase retarder as calculated by SRW with that of a true plane wave as predicted by dynamical diffraction theory 
and also with measurements taken at NSLS-II ISR. Figure 3 makes the same comparisons for a quasi-plane wave of $6844 \mathrm{eV}$ and the $240 \mu \mathrm{m}$ thick phase retarder. The agreement between the SRW simulation and plane-wave dynamical diffraction theory is excellent, as it should be. The measurements of the linearly vertically polarized intensity in part (d) of both figures are similar to the calculations in part (a), although the maxima do not appear at quite the expected angles. This already indicates that there are errors in the alignment of the phase retarder in the beamline, as will be discussed later.

Table 1. Thickness of the diamond symmetric (111) phase retarders used at NSLS-II ISR, photon energies, and radii of quasi-planar wavefront entering each phase retarder in the first benchmark.

\begin{tabular}{|l|l|l|}
\hline \multicolumn{1}{|c|}{ Thickness $(\boldsymbol{\mu m})$} & \multicolumn{1}{|c|}{ Photon energy (eV) } & \multicolumn{1}{c|}{$\begin{array}{c}\text { Radius of incident } \\
\text { wavefront }(\mathbf{m})\end{array}$} \\
\hline 510 & 8708 & $2.79 \times 10^{7}$ \\
\hline 240 & 6844 & $1.72 \times 10^{7}$ \\
\hline
\end{tabular}
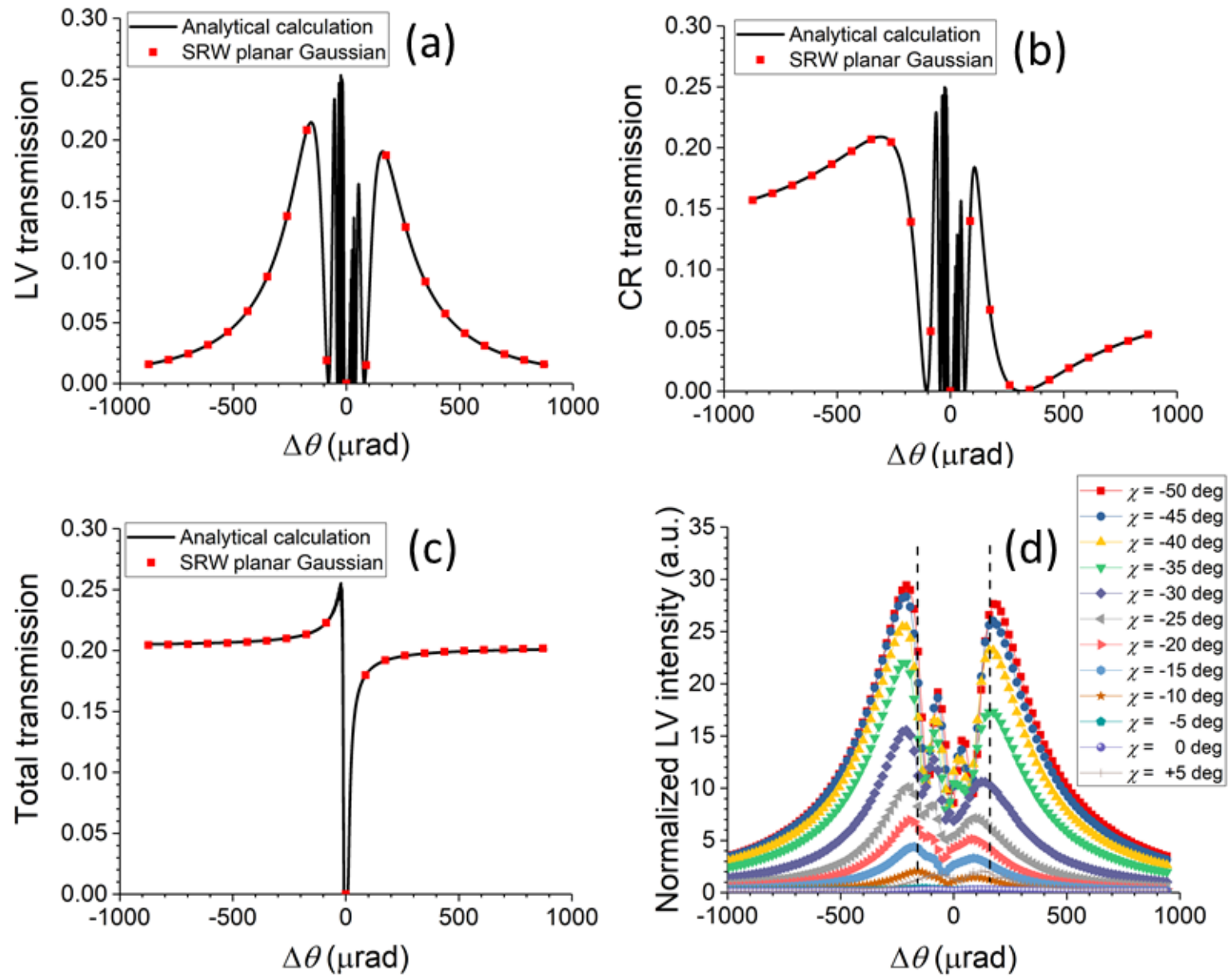

Figure 2. First benchmark: $510 \mu \mathrm{m}$ thick symmetric (111) diamond phase retarder in $8708 \mathrm{eV}$ X-rays. (a)-(c): Check of SRW simulation of phase retarder in quasi-plane wave (red squares) against plane-wave dynamical diffraction theory (black line). $\mathrm{LV}=$ linear vertical polarization, $\mathrm{CR}=$ right circular polarization. (d) Experimental measurements collected at the NSLS-II ISR beamline for the linearly vertically polarized beam downstream from the phase retarder. The vertical dashed lines mark the positions of the maxima in (a). The polarization analyzer used to separate out the linearly vertically polarized component was a LiF crystal oriented to the (400) Bragg reflection. The plots were recorded in steps of $5^{\circ}$ in $\chi$. 

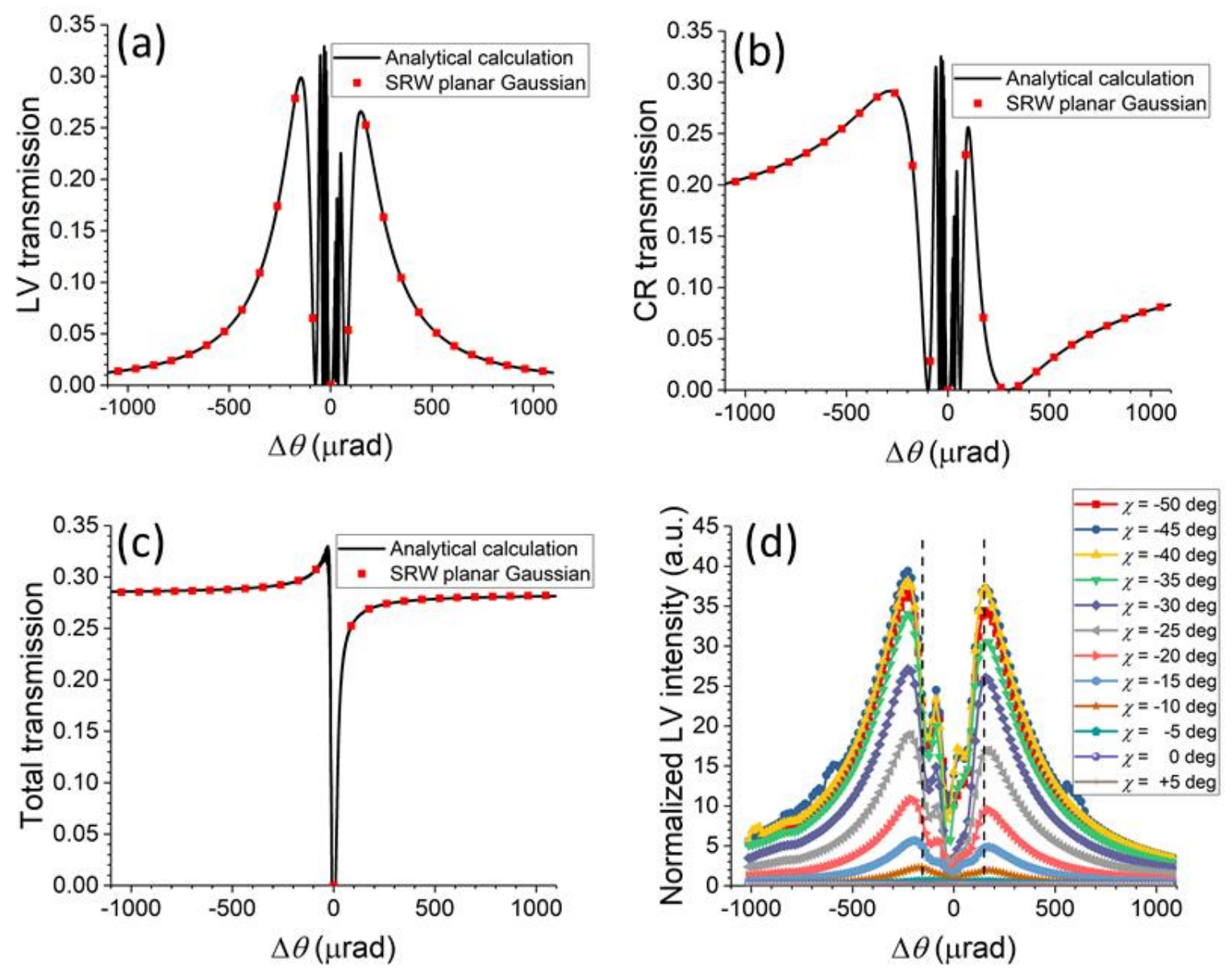

Figure 3. First benchmark: $240 \mu \mathrm{m}$ thick symmetric (111) diamond phase retarder in $6844 \mathrm{eV} \mathrm{X-rays.} \mathrm{(a)-(c):} \mathrm{Check} \mathrm{of}$ SRW simulation of phase retarder in quasi-plane wave (red squares) against plane-wave dynamical diffraction theory (black line). $\mathrm{LV}=$ linear vertical polarization, $\mathrm{CR}=$ right circular polarization. (d) Experimental measurements collected at the NSLS-II ISR beamline for the linearly vertically polarized beam downstream from the phase retarder. The vertical dashed lines mark the positions of the maxima in (a). The polarization analyzer used to separate out the linearly vertically polarized component was a $\mathrm{Cu}$ crystal oriented to the (220) Bragg reflection. The plots were recorded in steps of $5^{\circ}$ in $\chi$.

\subsection{Second benchmark: idealized version of NSLS-II ISR beamline}

Here only the $240 \mu \mathrm{m}$ thick phase retarder at $6844 \mathrm{eV}$ is examined. The optical layout of the NSLS-II ISR beamline is shown in Figure 4. The source is an ideal U23 undulator of $2.8 \mathrm{~m}$ length. Its gap is adjusted so that the desired photon energy falls on its third harmonic. The undulator radiation is linearly polarized in the horizontal direction. Only singleelectron radiation is considered here. The horizontally focusing mirror (HFM) is horizontally deflecting and tangentially curved. The grazing angle of incidence at its center is $2.618 \mathrm{mrad}$. Its dimensions are $1 \mathrm{~m}$ long and $0.1 \mathrm{~m}$ wide. The vertically focusing mirror (VFM) is vertically deflecting and tangentially curved. Its grazing angle of incidence and its dimensions are the same as those of the HFM. The double-crystal monochromator (DCM) is composed of two nondispersively arranged, vertically deflecting symmetric Si (111) crystals. The phase retarder is set to $\chi=45^{\circ}$. Thermal distortions and figure errors on the optical components are neglected.

Figure 5 compares the SRW simulations of the phase retarder's total and polarization-resolved transmission on the idealized NSLS-II ISR beamline with the analytical calculations of plane-wave dynamical diffraction theory. The total transmission calculated by SRW as a function of deviation from the Bragg angle tracks the plane-wave calculations very well. However, the minima and maxima of the linearly horizontally (LH) polarized and the linearly vertically (LV) polarized components are less prominent in the SRW simulations. The sharper these extrema are in the plane-wave 
calculations, the more severely they are weakened when the undulator beam is used. Also, at the edges of the total reflection region, where the plane-wave calculations show a rapid oscillation of the transmitted beam between $\mathrm{LH}$ and LV polarization, the SRW calculations with undulator beam show an averaged value for both polarizations. Figure 6 shows that this smearing of the angular dependence of the LH and LV polarization components results from the nonzero divergence of the undulator beam. Figure 6(a) displays the LV component of the wavefront $1 \mathrm{~m}$ downstream from the phase retarder on the idealized NSLS-II ISR beamline as simulated by SRW when $\theta$ is $-75 \mu \mathrm{rad}$ from the center of the total reflection region. At this point, Figure 5(c) shows that the LV transmission through the phase retarder reaches a minimum. The incomplete suppression of the LV transmission in the beamline simulation results from the leakage of the outer sections of the undulator beam. One may similarly see the leakage of the LH component of the outer parts of the undulator beam when $\theta$ is $-50 \mu \mathrm{rad}$ from the center of the total reflection region, where Figure 5(b) shows that the LH transmission reaches a minimum. Figure 6(c) and (d) show the spatial distribution of, respectively, the LH and LV components of the wavefront $1 \mathrm{~m}$ downstream from the phase retarder on the idealized NSLS-II ISR beamline when $\theta$ is $-20 \mu \mathrm{rad}$ from the center of the total reflection region. Here, the rapid oscillation of polarization with $\theta$ seen in Figure 5 (b) and (c) is mirrored in the fringes of the spatial distribution.

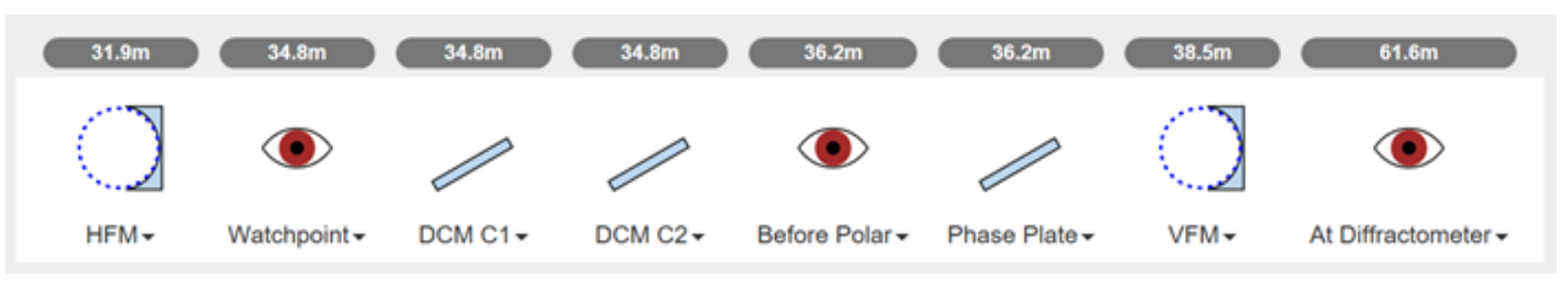

Figure 4. Optical layout of NSLS-II beamline. Copied from Sirepo interface ${ }^{12}$. HFM $=$ horizontally focusing mirror. DCM $=$ double-crystal monochromator. VFM = vertically focusing mirror.
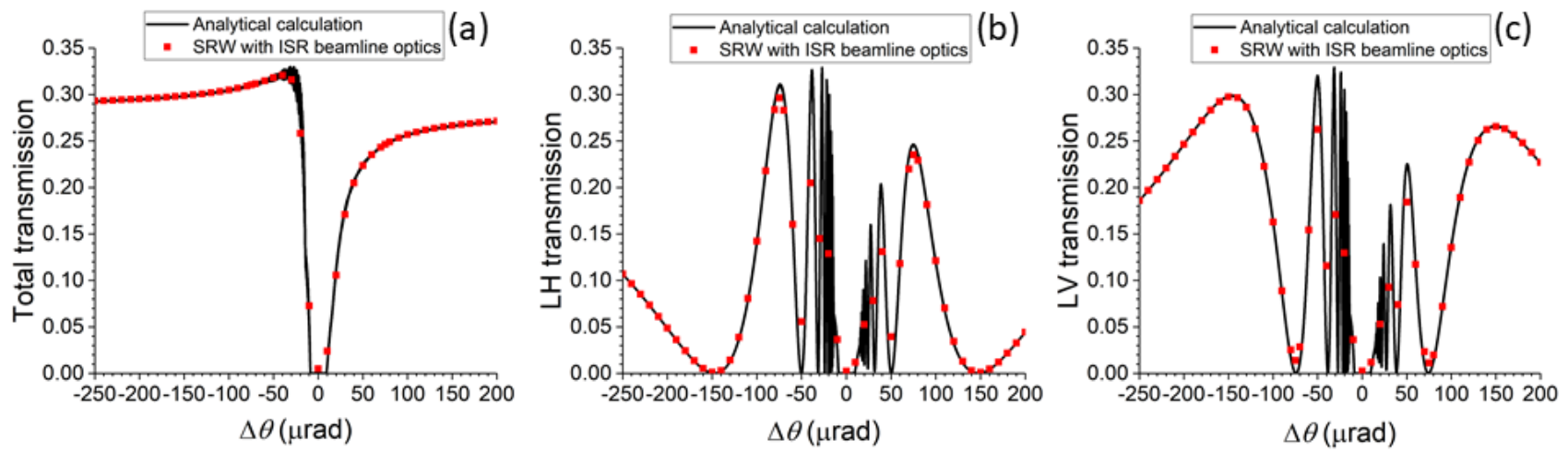

Figure 5. Second benchmark: SRW simulations of idealized NSLS-II ISR beamline (red squares) compared with analytical calculations from dynamical diffraction theory (black line). (a) Total transmission through phase retarder, including all polarization components. (b) Linearly horizontally (LH) polarized component of transmission through phase retarder. (c) Linearly vertically (LV) polarized component of transmission through phase retarder.

The focusing of the beam onto a sample may bring forth more subtle effects. Kato ${ }^{26}$ showed that the direction of the time- and space-averaged energy flow of the wavefields propagating in a perfect crystal near a Bragg reflection depends on the incidence angle of the X-ray beam. The energy flow will lie between the direction of the incident beam and that of the diffracted beam, and a $\mu$ rad rotation of the crystal relative to the incident beam may change the direction of the energy flow by several degrees. This would in principle cause a small displacement of the beam transmitted through the phase retarder as $\theta$ is varied to change the beam polarization. Figure 7 compares the position of the $\mathrm{LV}$ component of the beam at the focal spot for $\Delta \theta=-150 \mu \mathrm{rad}$, which is an LV maximum, and for $\Delta \theta=-100 \mu \mathrm{rad}$, which is close to the next $\mathrm{LV}$ minimum. The beam shifts from the upper left to the lower right by about $1 \mu \mathrm{m}$. This does not seem very large, but is still a significant fraction of the idealized FWHM focal spot size of $13.3 \mu \mathrm{m}$ horizontal $\times 8.7 \mu \mathrm{m}$ vertical. 

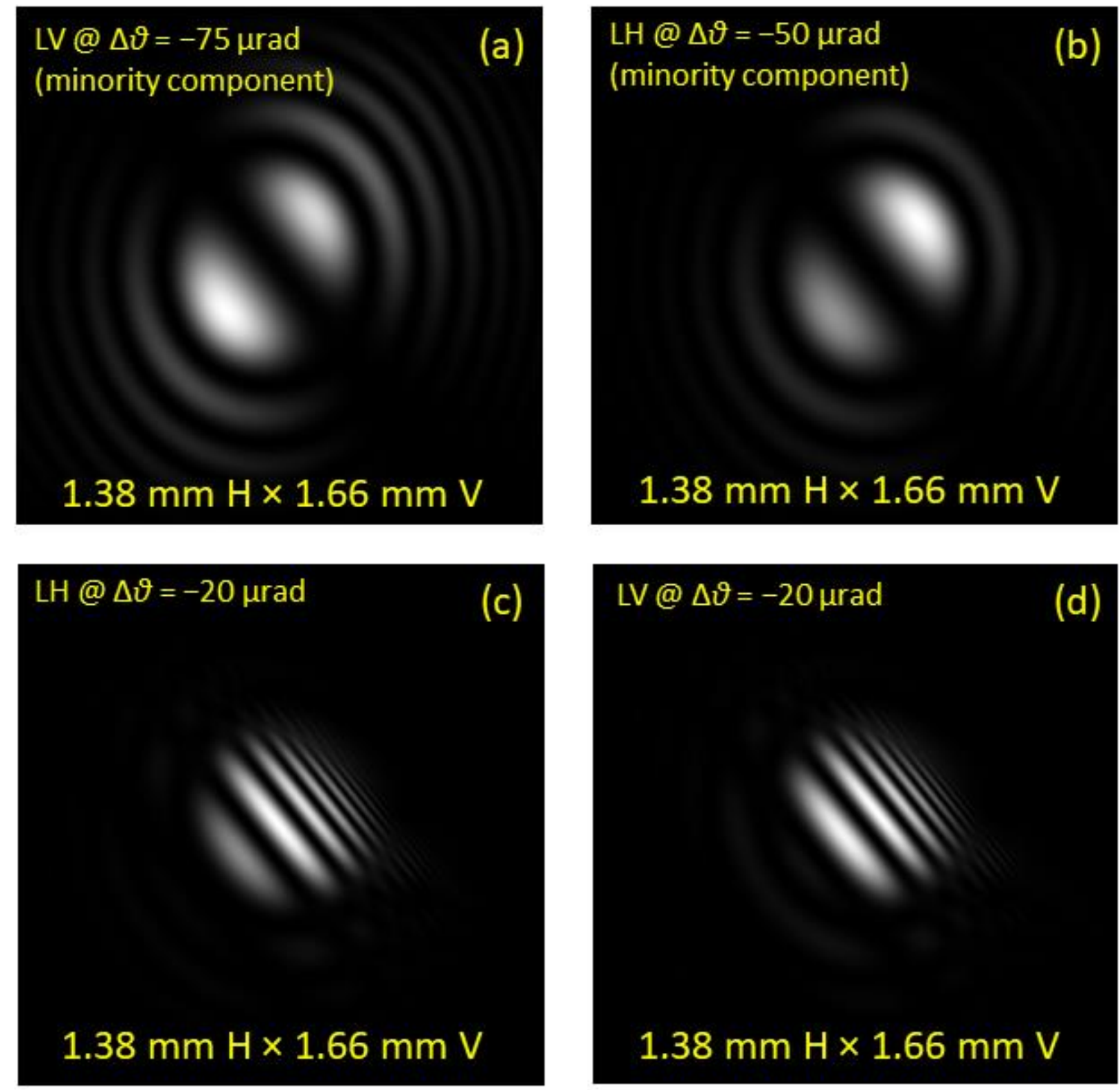

Figure 6. SRW simulations of spatial distribution of LH and LV components of single-electron wavefront at NSLS-II ISR beamline, $1 \mathrm{~m}$ downstream from the phase retarder. The field of view in each image is $1.38 \mathrm{~mm}$ horizontal $\times 1.66 \mathrm{~mm}$ vertical. (a) and (b) are taken, respectively, at a minimum for LV transmission and at a minimum for LH transmission. (c) and (d) are the LH and LV components when the phase retarder is oriented to the left edge of the total reflection region, where the polarization of the transmitted beam oscillates rapidly with incidence angle. 

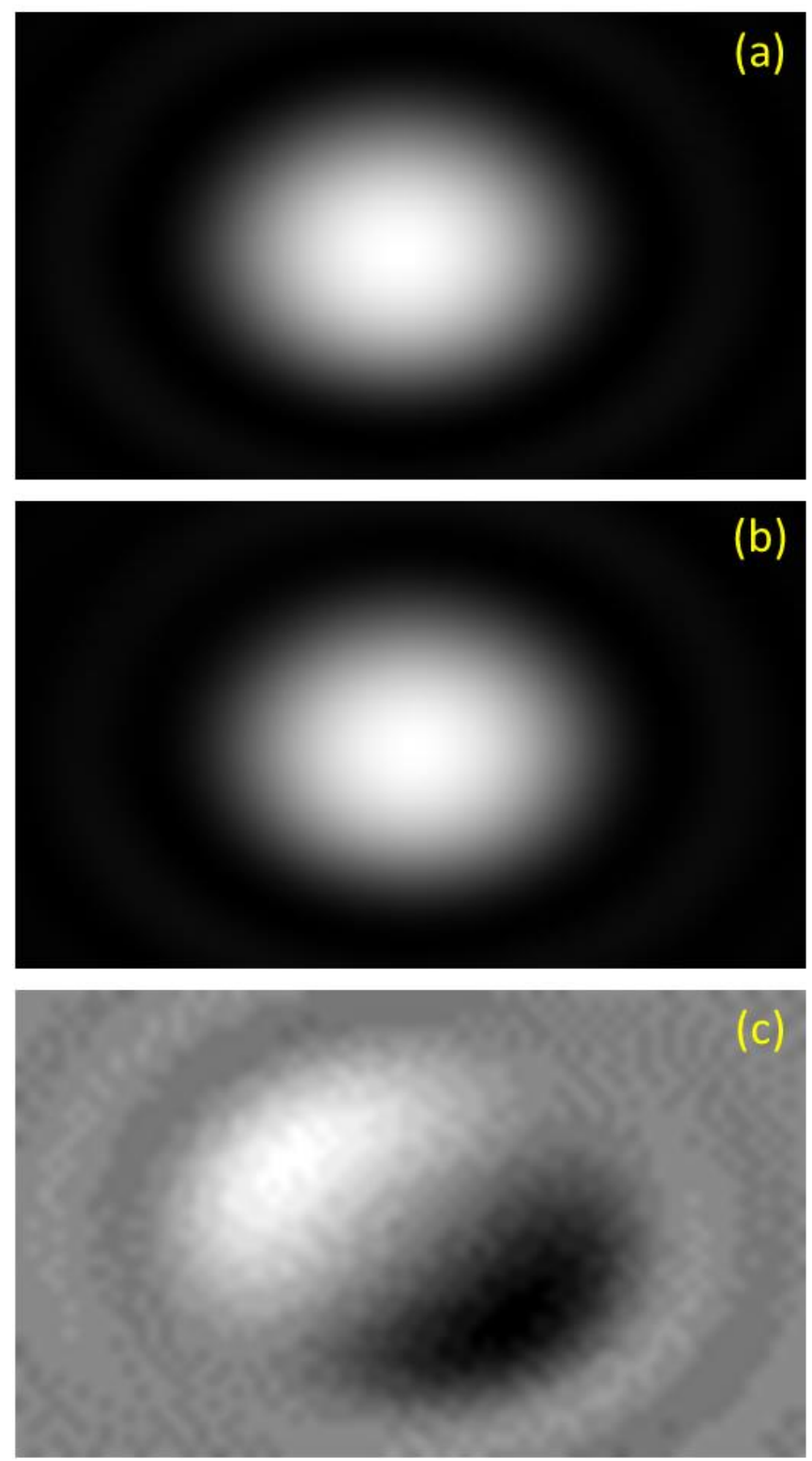

Figure 7. Spatial distribution of LV component of single-electron wavefront of idealized NSLS-II ISR at focal point (the sample) as calculated by SRW. (a) $\Delta \theta=-150 \mu \mathrm{rad}$. (b) $\Delta \theta=-100 \mu \mathrm{rad}$. (c) Image (a) - Image (b), showing the shift of about $1 \mu \mathrm{m}$ in the beam's position. The FWHM of the focal spot is $13.3 \mu \mathrm{m}$ horizontal $\times 8.7 \mu \mathrm{m}$ vertical. The field of view in each image is $38.2 \mu \mathrm{m}$ horizontal $\times 18.9 \mu \mathrm{m}$ vertical. 
Finally, one can examine whether the LV component of the focal spot changes in shape if $\Delta \theta$ is changed. Figure 8 shows the LV component of the idealized NSLS-II ISR beamline's focus at two LV maxima: $\theta=-150 \mu \mathrm{rad}$ and $\Delta \theta=-50 \mu \mathrm{rad}$. Figure 5(c) shows that the first maximum has a very gradual dependence on $\Delta \theta$, while the second is very sharp and thus is smeared out by the nonzero divergence of the focused undulator beam. The plots do in fact reveal that the profile for the second, sharper maximum is slightly narrower than the profile for the broader first maximum. The oscillations in the second maximum are also less prominent.
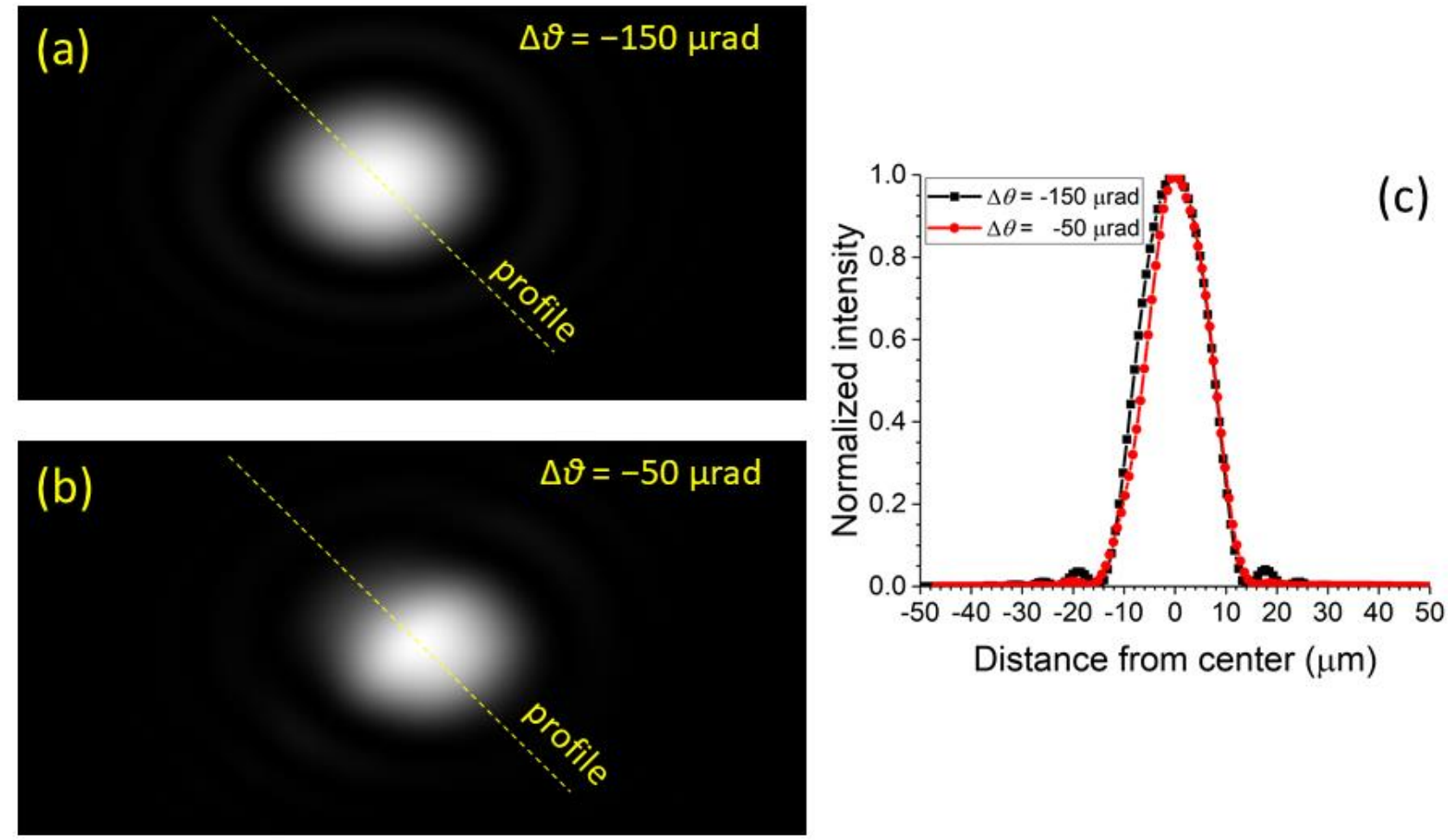

Figure 8. SRW simulation of LV component of focal spot at sample position of idealized NSLS-II ISR beamline at two LV maxima. (a) $\Delta \theta=-150 \mu \mathrm{rad}$ (b) $\Delta \theta=-50 \mu \mathrm{rad}$. (c) Profiles of both focal spots along dotted lines labelled "Profile" in (a) and (b).

\subsection{Comparison of SRW with experimental data}

The experimental apparatus used to vary the beam polarization and measure its LV component on the NSLS-II ISR beamline is shown in Figure 9. In order to minimize air absorption at lower photon energies, the beam from the phase retarder is sent through an evacuated flight path on its way to the analyzer crystal, which deflects the beam horizontally with a Bragg angle of $45^{\circ}$. At $8708 \mathrm{eV}$, the analyzer is a LiF crystal oriented to the symmetric (400) Bragg reflection. At $6844 \mathrm{eV}$, the analyzer is a $\mathrm{Cu}$ crystal oriented to the symmetric (220) Bragg reflection. This analyzer suppresses the LH component of the beam from the phase retarders, leaving only the LV component to be passed onward to the avalanche photodiode detector. The intensity recorded by the avalanche photodiode is normalized to the total transmitted intensity recorded by an ion chamber between the phase retarder and the polarization analyzer.

As before, for this paper, the $240 \mu \mathrm{m}$ thick phase retarder at $6844 \mathrm{eV}$ is examined. Figure 10(a) displays a series of experimental scans in $\theta$ of the $\mathrm{LV}$ component transmitted by the phase retarder at values of $\chi$ from $-50^{\circ}$ to $+5^{\circ}$. Figure 10(b) shows a series of theoretical LV scans in $\theta$ at various values of $\chi$ as predicted by plane-wave dynamical diffraction. The experimental scans look similar to the theoretical calculations, but close examination reveals a subtle discrepancy: while the positions of the LV maxima are independent of $\chi$ in the theoretical calculations, the left-hand LV maximum in the experimental data shifts progressively toward the center of the rocking curve as $\chi$ increases beyond $-25^{\circ}$. 

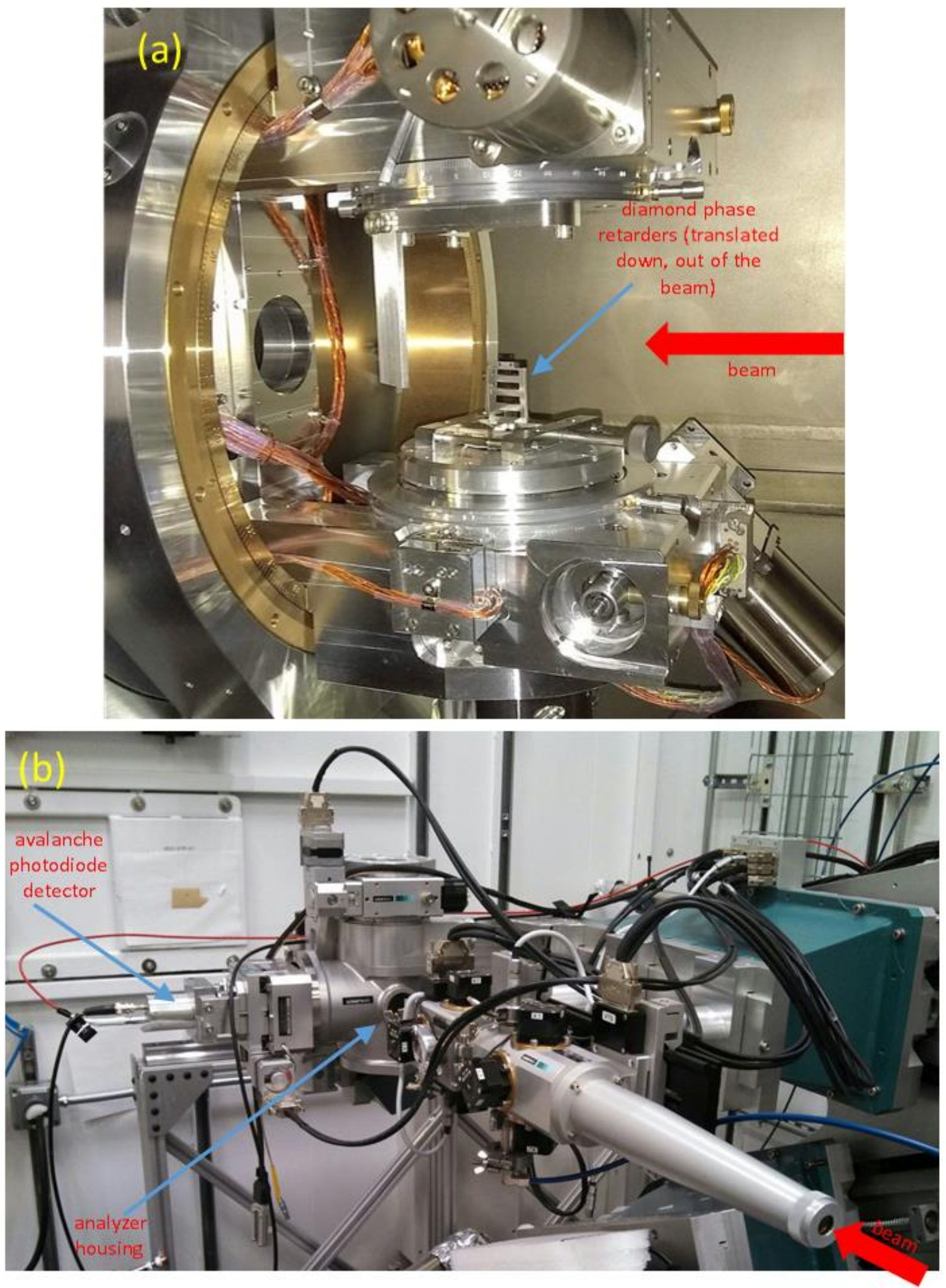

Figure 9. Photographs of polarization-modifying apparatus on the NSLS-II ISR beamline. (a) Diamond phase retarders mounted on a Huber chi circle. (b) Assembly of analyzer and detector mounted on $2 \theta$ arm of Huber 6-circle diffractometer. 
Furthermore, one would naively expect the LV intensity at the maxima to have a $\sin ^{2}(2 \chi)$ dependence. This is borne out by Figure 11(a), which shows the plane-wave dynamical diffraction calculation, and Figure 11(b), which provides the SRW simulation on the idealized NSLS-II ISR beamline. However, the dependence of the experimental LV maxima on $\sin ^{2}(2 \chi)$, plotted in Figure 11(c), is imperfect. One possible cause, which remains to be investigated, is that the length of the beam path through the phase retarder does not remain constant as $\chi$ is varied because the phase retarder is misaligned in the beam.
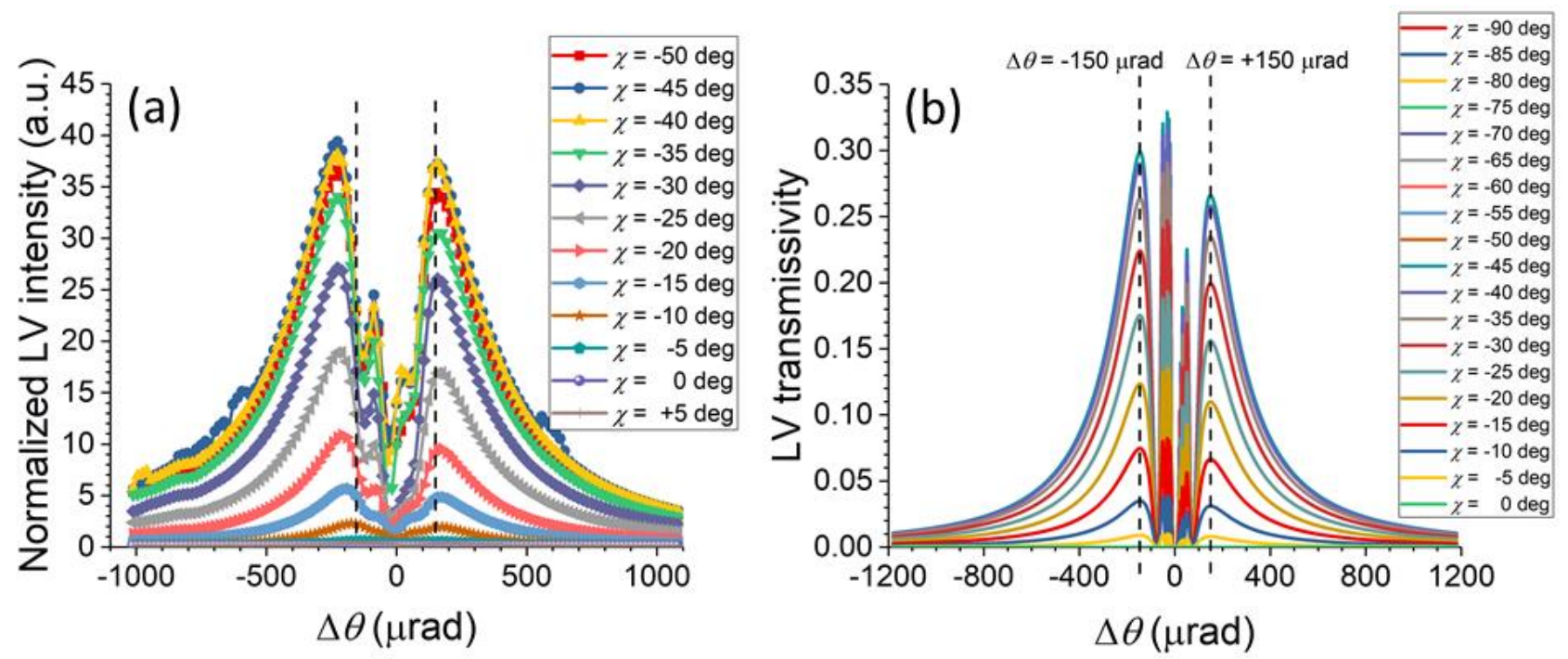

Figure 10. Scans in $\theta$ at various values of $\chi$. (a) Experimental measurements of normalized LV intensity downstream from $240 \mu \mathrm{m}$ thick phase retarder (same as in Figure 3(d)). (b) Plane-wave analytical calculations of the ideal LV transmissivity.
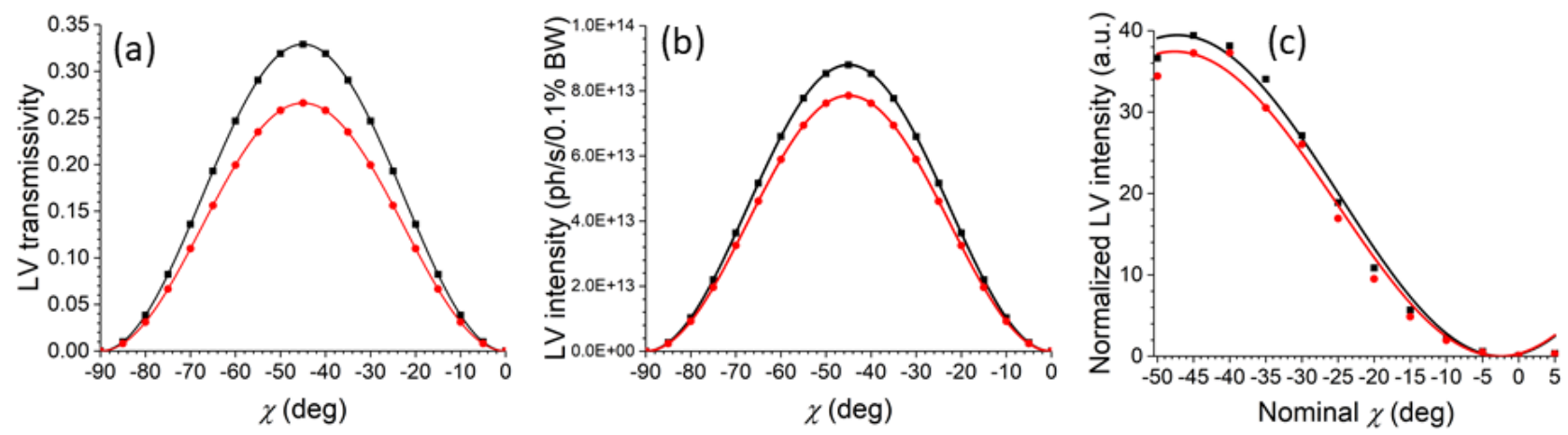

Figure 11. (a) Fits to $\sin ^{2}(2 \chi)$ of theoretical LV transmissivity of phase retarder calculated by plane-wave dynamical diffraction at the left-hand (black squares) and right-hand (red circles) LV maxima. Solid curves are the best fits. (b) Fits to $\sin ^{2}(2 \chi)$ of theoretical LV intensity downstream from phase retarder calculated by SRW on the idealized NSLSII ISR beamline at the left-hand (black squares) and right-hand (red circles) LV maxima. Solid curves are the best fits. (c) Fits to $\sin ^{2}(2 \chi+\varphi)$ of experimental measurements of LV intensity from phase retarder at the left-hand (black squares) and right-hand (red circles) LV maxima. Solid curves are the best fits.

\section{CONCLUSIONS}

The perfect-crystal X-ray Bragg reflection propagator that was already a standard part of the SRW toolbox now also treats transmission in the Bragg geometry. This Bragg transmission propagator has been benchmarked with simulations of an ideal phase retarder that diffracts either quasi-plane waves or a single-electron undulator wavefront focused with ideal mirrors. Experimental data are similar to the SRW simulations, but with slight discrepancies that may be explainable by a misalignment of the phase retarder in the beam. The calculations of this paper allow the tolerances of the phase retarder's angular orientation to be determined. Most importantly, Bragg-transmission optical components at 
real synchrotron and XFEL beamlines, which have nonzero electron beam emittances, various types of undulators, and imperfect focusing optics, can be designed in SRW with greater capability and confidence.

\section{ACKNOWLEDGEMENTS}

This research used beamline 4-ID of the National Synchrotron Light Source II, a U.S. Department of Energy (DOE) Office of Science User Facility operated for the DOE Office of Science by Brookhaven National Laboratory under Contract No. DE-SC0012704. The development of SRW and Sirepo was supported by DOE Contract No. DESC0011237 and DOE BES Field Work Proposal PS-017. The authors thank Diamond Light Source for its longstanding support and David Laundy of that institute for fruitful discussions.

\section{REFERENCES}

[1] Zachariasen, W. H., [Theory of X-ray Diffraction in Crystals], John Wiley \& Sons, New York (1945).

[2] Batterman, B. W. and Cole, H., "Dynamical diffraction of X rays by perfect crystals," Rev. Mod. Phys. 36(3), 681-717 (1964).

[3] Authier, A., [Dynamical Theory of X-Ray Diffraction], International Union of Crystallography, Oxford Science Publications (2001).

[4] Shvyd'ko, Y., "Dynamical Theory of X-ray Diffraction (Focus on backscattering)," in [X-Ray Optics], Springer Series in Optical Sciences, Vol. 98, Springer, Berlin \& Heidelberg (2004).

[5] Knudsen, E. B., Prodi, A., Baltser, J., Thomsen, M., Willendrup, P. K., Sanchez del Rio, M., Ferrero, C., Farhi, E., Haldrup, K., Vickery, A., Feidenhans'l, R., Mortensen, K., Nielsen, M. M., Poulsen, H. F., Schmidt, S. and Lefmann, K. "McXtrace: a Monte Carlo software package for simulating X-ray optics, beamlines and experiments," J. Appl. Cryst. 46(3), 679-696 (2013).

[6] Schäfers, F., "The BESSY Raytrace Program RAY," in [Modern Developments in X-Ray and Neutron Optics], Erko, A., Idir, M., Krist, T. and Michette, A. G., eds., Springer Series in Optical Sciences, Vol. 137, Springer, Berlin \& Heidelberg (2008).

[7] Sanchez del Rio, M., Canestrari, N., Jiang, F. and Cerrina, F., "SHADOW3: a new version of the synchrotron X-ray optics modeling package," J. Synchrotron Rad. 18(5), 708-716 (2011).

[8] Bahrdt, J., Flechsig, U., Gerhardt, S. and Schneider, I., "PHASE, a universal software package for the propagation of time-dependent coherent light pulses along grazing incidence optics," Proc. SPIE 8141, 81410E (2011).

[9] Flechsig, U., Bahrdt, J., Follath, R. and Reiche, S. "Physical optics simulations with PHASE for SwissFEL beamlines," AIP Conf. Proc. 1741, 040040 (2016).

[10] Chubar, O. and Elleaume, P., "Accurate and efficient computation of synchrotron radiation in the near field region," in [Proceedings of EPAC '98, Sixth European Particle Accelerator Conference], Liljeby, L, Myers, S., Petit-Jean-Genaz, C., Poole, J. and Rensfelt, K.-G., eds., 1177-1179 (1998).

[11] Sutter, J. P., Chubar, O. and Suvorov, A., "Perfect crystal propagator for physical optics simulations with 'Synchrotron Radiation Workshop,'” Proc. SPIE 9209, 92090L (2014).

[12] Rakitin, M. S., Moeller, P., Nagler, R., Nash, B., Bruhwiler, D. L., Smalyuk, D., Zhernenkov, M. and Chubar, O., "Sirepo: an open-source cloud-based software interface for X-ray source and optics simulations," J. Synchrotron Rad. 25(6), 1877-1892 (2018).

[13] Sanchez del Rio, M. and Rebuffi, L., "OASYS: A software for beamline simulations and synchrotron virtual experiments," AIP Conf. Proc. 2054, 060081 (2019).

[14] Sutter, J. P., Ishikawa, T., Kuetgens, U., Materlik, G., Nishino, Y., Rostomyan, A., Tamasaku, K. and Yabashi, M., “An X-ray BBB Michelson interferometer,” J. Synchrotron Rad. 11(5), 378-385 (2004).

[15] Shvyd'ko, Y., Stoupin, S., Shu, D., Collins, S. P., Mundboth, K., Sutter, J. and Tolkiehn, M., "High-contrast sub-millivolt inelastic X-ray scattering for nano- and mesoscale science," Nat. Commun. 5, 4219 (2014).

[16] Molière, G. "Quantenmechanische Theorie der Röntgenstrahlinterferenzen in Kristallen," Ann. Phys. (Leipzig), 5. Folge, 35, 272-313 (1939).

[17] Baranova, N. B. and Zel'dovich, B. Ya., "Birefringence and gyrotropy due to nearly Bragglike processes in the X-ray region," Sov. Phys. JETP 52 (5), 900-904 (1980). 
[18] Skalicky, P. and Malgrange, C., "Polarization phenomena in X-ray diffraction," Acta Cryst. A28(6), 501-507 (1972).

[19] Golovchenko, J. A., Kincaid, B. M., Levesque, R. A., Meixner, A. E., and Kaplan, D. R., "Polarization Pendellösung and the generation of circularly polarized X rays with a quarter-wave plate," Phys. Rev. Lett. 57(2), 202-205 (1986).

[20] Giles, C., Malgrange, C., Goulon, J., de Bergevin, F. and Vettier, C., "Energy-dispersive phase plate for magnetic circular dichroism experiments in the X-ray range,” J. Appl. Cryst. 27(3), 232-240 (1994).

[21] Lang, J. C. and Srajer, G., "Bragg transmission phase plates for the production of circularly polarized X rays," Rev. Sci. Instrum. 66(2), 1540-1542 (1995).

[22] Waasmaier, D. and Kirfel, A., "New analytical scattering-factor functions for free atoms and ions," Acta Cryst. A51(3), 416-431 (1995).

[23] Chantler, C. T., Olsen, K., Dragoset, R. A., Chang, J., Kishore, A. R., Kotochigova, S. A. and Zucker, D. S. [XRay Form Factor, Attenuation and Scattering Tables (version 2.1)] Online. Available: http://physics.nist.gov//ffast. National Institute of Standards and Technology, Gaithersburg, Maryland, USA (2005).

[24] Schoening, F. R. L. and Vermeulen, L. A., "X-ray measurement of the Debye temperature for diamond at low temperatures," Solid State Commun. 7(1), 15-18 (1969).

[25] Stoupin, S. and Shvyd'ko, Y. V., "Ultraprecise studies of the thermal expansion coefficient of diamond using backscattering X-ray diffraction," Phys. Rev. B83(10), 104102 (2011).

[26] Kato, N., "The flow of X-rays and material waves in ideally perfect single crystals," Acta Cryst. 11, 885-887 (1958). 